\title{
Plasticity Detection and Quantification in Monopile Support Structures Due to Axial Impact Loading
}

\author{
P.C. Meijers ${ }^{1}{ }^{\star}, A$. Tsouvalas ${ }^{1}$, and A.V. Metrikine ${ }^{1}$ \\ ${ }^{1}$ Dept. of Hydraulic Engineering, Delft University of Technology, the Netherlands
}

\begin{abstract}
Recent developments in the construction of offshore wind turbines have created the need for a method to detect whether a monopile foundation is plastically deformed during the installation procedure. Since measurements at the pile head are difficult to perform, a method based on measurements at a certain distance below the pile head is proposed in this work for quantification of the amount of plasticity. By considering a onedimensional rod model with an elastic-perfectly plastic constitutive relation, it is shown that the occurrence of plastic deformation caused by an impact load can be detected from these measurements. Furthermore, this plastic deformation can be quantified by the same measurement with the help of an energy balance. The effectiveness of the proposed method is demonstrated via a numerical example.
\end{abstract}

\section{Introduction}

In recent years, the number of offshore wind farms has considerably increased to meet the demand for energy from renewable resources. The most commonly used foundation concept in offshore wind is a steel monopile [1]. Steel monopiles are thin-walled cylindrical structures that are normally driven into the seabed with a hydraulic impact hammer. Each hammer blow induces stress waves in the pile which help the latter to gradually progress into the soil.

As soon as the pile reaches the desired penetration depth, the wind turbine is installed on top of the support structure. Until recently, grouted connections were used between the foundation pile and the superstructure, however, bolted connections have become increasingly popular in recent years [1]. Compared to a grouted connection, a bolted connection is more sensitive to material damage inflicted by the pile driving process since the wind turbine is directly connected to the pile head. In addition, material damage in the pile reduces the service life of the support structure. A method to detect and quantify the damage caused by the pile driving process is therefore essential in order to assess the structural health of a monopile.

Measuring forces and deformations directly at the pile head is a cumbersome task not only due to the high stress levels expected at this critical location, but, additionally, due to the practical implementation of the sensors very close to the pile head. Furthermore, sensors at this location need careful installation to withstand high strains, which is difficult given the hard conditions at sea and the tight working schedules. Thus, a plasticity detection method based on acceleration or strain measurements a few meters

\footnotetext{
^e-mail: p.c.meijers@tudelft.nl
}

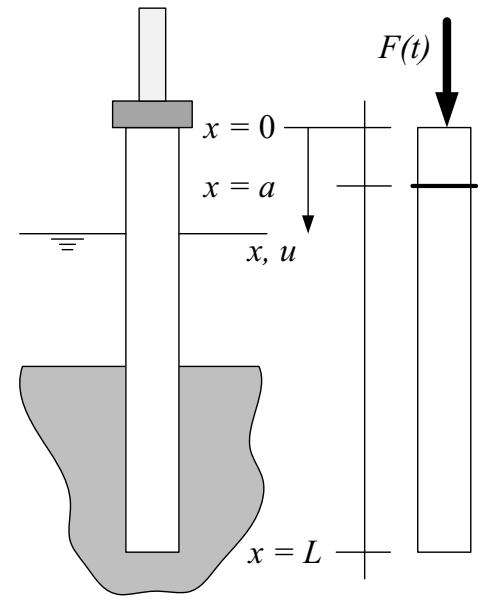

Figure 1: Sketch of the pile driving process (left) and the mathematical one-dimensional model (right).

below the pile head seems more feasible. Currently, sensors at this location are routinely used for the analysis of the driving process [2]. Given that, the proposed method of quantification of plasticity fits well within the current practice of the offshore industry. This work focuses on using the measurement data from these sensors to detect and quantify plastic deformations inflicted by the pile driving process.

\section{Model}

A sketch of the pile driving process is shown in Figure 1. The monopile is modelled by the one-dimensional rod theory [4], which includes the Rayleigh-Love correction term [5] to account for stress wave dispersion expected for 


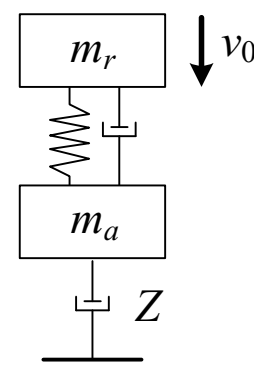

Figure 2: The hammer model of Deeks and Randolph. [3]

piles of large diameter. The model relates the axial displacement $u(x, t)$ and the axial stress $\sigma(x, t)$ as follows:

$$
\begin{aligned}
\rho \frac{\partial^{2} u}{\partial t^{2}} & =\frac{\partial \sigma}{\partial x}, \\
\varepsilon & =\frac{\partial u}{\partial x}, \\
\sigma & =E\left(\varepsilon-\varepsilon_{p}\right)+\underbrace{v^{2} r_{g}^{2} \frac{\partial^{2} \sigma}{\partial x^{2}}}_{\text {RL-correction }},
\end{aligned}
$$

in which $\rho, E$ and $v$ are the density, Young's modulus and Poisson's ratio, respectively. The radius of gyration of the cross-section is denoted by $r_{g}$ and the total axial strain by $\varepsilon$. To account for plastic deformation in the equations above, auxiliary relations for the plastic axial strain, $\varepsilon_{p}(x, t)$, are needed. Assuming perfect plasticity, these relations read [6]:

$$
\begin{gathered}
\frac{\partial \varepsilon_{p}}{\partial t}=\gamma \operatorname{sign}(\sigma), \\
f(\sigma)=|\sigma|-\sigma_{y} \leq 0, \\
\gamma \geq 0, \quad f(\sigma) \leq 0, \quad \gamma f(\sigma)=0, \\
\gamma \frac{\partial f(\sigma)}{\partial t}=0 \quad \text { if } f(\sigma)=0,
\end{gathered}
$$

where $\gamma$ is the magnitude of the plastic flow rate, $f(\sigma)$ the yield function and $\sigma_{y}$ the yield stress of the material.

The hammer force is described in this model by a time signal, which can be generated by a hammer model as shown in Figure 2 [3]. The time signal and the corresponding amplitude spectrum of the exerted hammer force obtain by a linear pile model are presented in Figure 3 with the dashed-dotted line. The input energy of the hammer is governed by the impact velocity of the ram $v_{0}$.

\section{Detection}

To show the effect of the non-linear material behaviour on the shape of the stress wave, simulated time signals of strain measurements at $x=a$ are considered. The measurement location is indicated in Figure 1 and it is assumed that no plastic deformation occurs at this crosssection. Figure 3 shows the strain signals of an elastic and an elasto-plastic model of the pile. Both cases are simulated with the same force signal as input. In the elastic

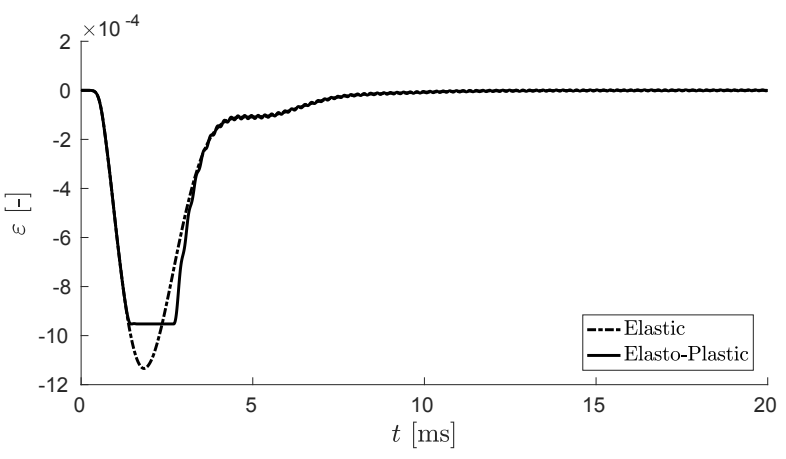

(a) Time signals.

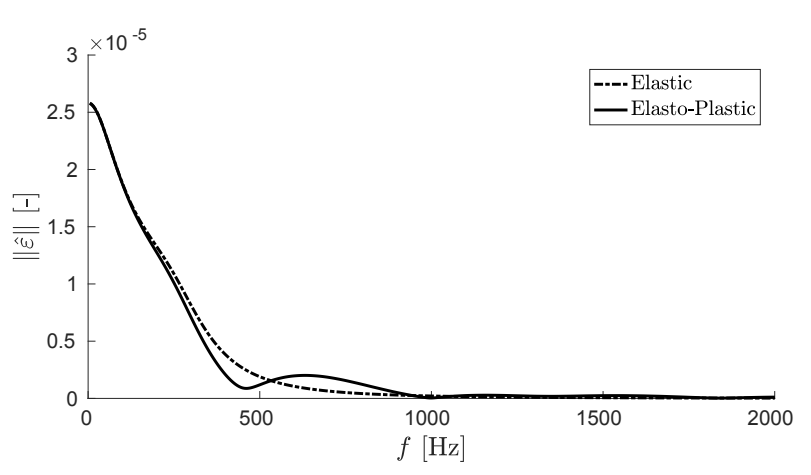

(b) Amplitude spectra.

Figure 3: Simulated strain signals at $x=a$ for an elastic and an elasto-plastic pile.

case, the time signal and the amplitude spectrum have the same shape as the input signal. However, a plateau and an elongation of the peak value in the strain time signal are seen for the elasto-plastic case. Furthermore, a shift in frequency content compared to the elastic case is observed. This shift is caused by the non-linear material behaviour. Thus, by comparing the measured strain signal with the expected strain computed on the basis of the elastic model of the structure, one can reach some preliminary conclusions regarding plastic deformation at the pile head.

\section{Quantification}

In order to quantify the plastic deformation, the energy balance is considered

$$
E_{0}=E_{w}+E_{p}+E_{\mathrm{loss}},
$$

where $E_{0}$ is the input energy of the hammer, $E_{w}$ the wave energy that passed through the considered cross-section, $E_{p}$ the energy lost in plastically deforming the material and $E_{\text {loss }}$ denotes the losses in sound radiation and material damping. Each contribution to the energy balance is now further discussed.

The input energy, $E_{0}$, is determined by the aforementioned hammer model [3] or by scaling the linear response of a low energy impact. The energy that passed through 


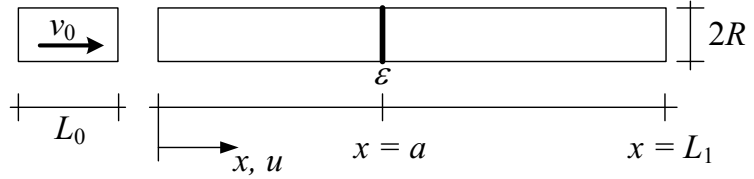

Figure 4: The modelled set-up of the experiment of Kolsky and Douch. [7]

the cross-section, $E_{w}$, is defined as the integral of the energy flux over time,

$$
E_{w}=\int_{t=t_{0}}^{t=t_{1}} F v \mathrm{~d} t
$$

which can be rewritten in terms of axial strain $\varepsilon$, using the relations $F=A \sigma, \sigma=E \varepsilon$ and $v=\frac{\sigma}{\sqrt{E \rho}}$ resulting in

$$
E_{w}=\frac{A E^{2}}{\sqrt{E \rho}} \int_{t=t_{0}}^{t=t_{1}} \varepsilon^{2} \mathrm{~d} t,
$$

in which $A$ is the area of the cross-section. The plastic work, $E_{p}$, is defined as the area under the stress-plastic strain diagram integrated over the distance between the impact and the sensor location,

$$
E_{p}=A \int_{x=0}^{x=a} \int_{\varepsilon=0}^{\varepsilon=\varepsilon_{p}} \sigma_{y} \mathrm{~d} \varepsilon \mathrm{d} x=A \sigma_{y} \int_{x=0}^{x=a} \varepsilon_{p} \mathrm{~d} x .
$$

The evaluation of the integral of $\varepsilon_{p}$ over $x$ gives the total plastic deformation $u_{p}$. This results in the final expression

$$
E_{p}=A \sigma_{y} u_{p} .
$$

Provided that the other losses in the pile, $E_{\text {loss }}$, are negligibly small, the permanent axial displacement, $u_{p}$, can be computed with

$$
u_{p}=\frac{E_{0}-E_{w}}{A \sigma_{y}} .
$$

This equation gives a simple relation to quantify the plastic deformation caused by an impact load based on a strain measurement at a certain distance from the impact point. Naturally, it is expected that this expression will give an upper bound estimation of the expected plastic strain due to the fact that $E_{\text {loss }}$ is assumed equal to zero.

\section{Numerical example}

As an example of the proposed method, the experiments of Kolsky and Douch [7] are considered. In their paper, a cylindrical copper specimen of length $L_{0}$ is impacted with initial velocity $v_{0}$ onto a stationary cylindrical copper rod with length $L_{1}$ as shown in Figure 4. Both the specimen and the rod have the same diameter. Dimensions and material properties are listed in Table 1 . From the initial velocity $v_{0}=14.02 \mathrm{~m} / \mathrm{s}(=46 \mathrm{ft} / \mathrm{s})$, it follows that the initial energy $E_{0}=1 / 2 m v_{0}^{2}=36.8 \mathrm{~J}$.

Since only the shape of the resulting stress wave is reported in [7], the stress wave propagation is simulated using the model introduced in Section 2. Equations 1 are
Table 1: Parameters used for the simulation of the experiment of Kolsky and Douch. [7]

\begin{tabular}{cl}
\hline Parameter & Value \\
\hline$L_{0}$ & $0.1524 \mathrm{~m}$ \\
$L_{1}$ & $1.2192 \mathrm{~m}$ \\
$R$ & $9.525 \mathrm{~mm}$ \\
$a$ & $0.5476 \mathrm{~m}$ \\
$E$ & $129.8 \mathrm{GPa}$ \\
$v$ & 0.34 \\
$\rho$ & $8960 \mathrm{~kg} / \mathrm{m}^{3}$ \\
$\sigma_{y}$ & $210 \mathrm{MPa}$ \\
\hline
\end{tabular}

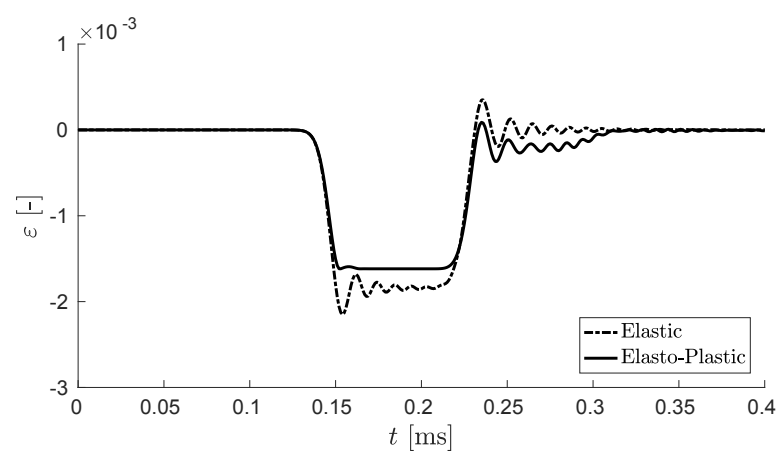

(a) Time signals.

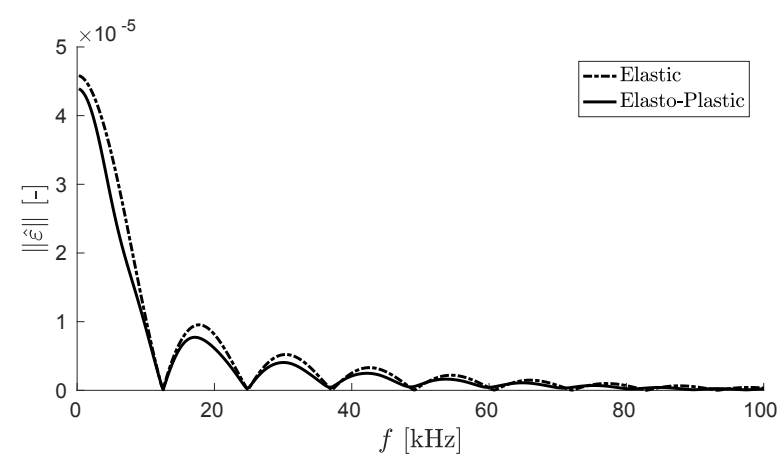

(b) Amplitude spectra.

Figure 5: Simulated strain signals at $x=0.548 \mathrm{~m}$ for the elastic and the elasto-plastic rod.

spatially discretised with the Finite Element Method in FEniCS [8] and an explicit Newmark scheme [9] is used for the time integration. The spatial resolution and time step are $\Delta x=2.74 \cdot 10^{-3} \mathrm{~m}$ and $\Delta t=1.0 \cdot 10^{-7} \mathrm{~s}$, respectively. Equations 2 are satisfied with the help of a return mapping algorithm [6].

Axial strain signals for the elastic and the elasto-plastic simulation at $x=0.548 \mathrm{~m}$ are shown in Figure 5. Just as reported in [7], a plateau and a tail are present in the shape of the stress wave for the elasto-plastic case, which indicates that plastic deformation occurred. By integrating the square of the strain signal, the energy that passed the cross-section is determined: $E_{w} \approx 29.0 \mathrm{~J}$. With the help of Equation 6, the permanent deformation computed equals $u_{p}=0.130 \mathrm{~mm}$. 


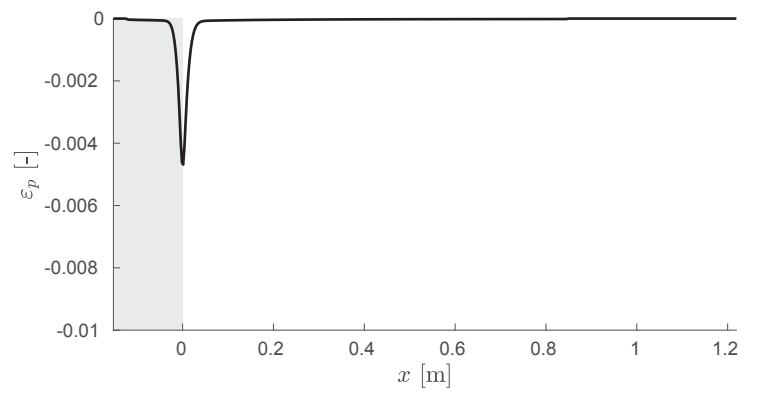

Figure 6: Plastic axial strain along the length of the rod. The shaded grey area is the length of the impact specimen $L_{0}$.

From the simulation, the permanent deformation can also be determined by integrating the plastic strain along the length of the rod up to the location of the sensor. The plastic strain profile is presented in Figure 6. Integrating the plastic strain results in $u_{p}=\int \varepsilon_{p} \mathrm{~d} x=0.131 \mathrm{~mm}$, which is in good agreement with the value found using the wave energy. This simple example shows how the plastic deformation can be quantified with the proposed method.

\section{Conclusions and future work}

By considering a one-dimensional rod model with an elastic-perfectly plastic constitutive relation, it is shown that the occurrence of plastic deformation caused by an impact load can be detected from a local measurement a certain distance from the impact point, since the shape of the stress wave is changed by the physically non-linear behaviour of the system. Furthermore, the plastic deformation can be quantified by the same measurement with the help of an energy balance, relating the input energy and the wave energy to the amount of permanent axial displacement. A numerical example simulating an impact experiment shows the effectiveness of the proposed plasticity detection and quantification method.

Future work will focus on generalising the constitutive relation to incorporate hardening behaviour and on im- proving the modelling of the monopile with a cylindrically symmetrical shell theory which will also include hammerpile interaction.

\section{Acknowledgements}

This research is part of the EUROS programme, which is supported by NWO domain Applied and Engineering Sciences and partly funded by the Dutch Ministry of Economic Affairs.

\section{References}

[1] WindEurope, Tech. rep., WindEurope (2017), The European Offshore Wind Industry. Key Trends and Statistics 2016

[2] F. Rausche, M. Nagy, S. Webster, L. Liang, CAPWAP and Refined Wave Equation Analyses for Driveability Predictions and Capacity Assessment of Offshore Pile Installations, in ASME 2009 28th International Conference on Ocean, Offshore and Arctic Engineering (American Society of Mechanical Engineers, 2009), pp. 375-383

[3] A.J. Deeks, M.F. Randolph, International Journal for Numerical and Analytical Methods in Geomechanics 17, 279 (1993)

[4] G.P. DeVault, Journal of the Mechanics and Physics of Solids 13, 55 (1965)

[5] K.F. Graff, Wave Motion in Elastic Solids (Dover Publications, Mineola, N. Y., 1991)

[6] J.C. Simo, T.J. Hughes, Computational Inelasticity, Vol. 7 (Springer Science \& Business Media, 2006)

[7] H. Kolsky, L.S. Douch, Journal of the Mechanics and Physics of Solids 10, 195 (1962)

[8] A. Logg, K.A. Mardal, G.N. Wells et al., Automated Solution of Differential Equations by the Finite Element Method (Springer, 2012), ISBN 978-3-64223098-1

[9] N.M. Newmark, Journal of the Engineering Mechanics Division 85, 67 (1959) 